\title{
High-Speed and High-efficient Modulo (2n-3) Multipliers
}

\author{
Liu Huihua* \\ School of Electronic Engineering \\ UESTC \\ Chengdu, China \\ lhhua@uestc.edu.cn
}

\author{
Zhou Wanting \\ Research Institute of Electronic Science and Technology \\ UESTC \\ Chengdu, China \\ zhouwt@uestc.edu.cn
}

\author{
Li Lei \\ Research Institute of Electronic Science and Technology \\ UESTC \\ Chengdu, China \\ leilee@uestc.edu.cn
}

\begin{abstract}
In this paper, an algorithm for designing efficient modulo $2 \mathrm{n}-3$ multipliers is proposed. With this algorithm, we can design the fastest among all known modulo 2n-3 multipliers by applying some simple correction terms. Implemented using 90nm CMOS process technology, the proposed modulo $2 \mathrm{n}-3$ multiplier can improve the current state of the art by 3.9\% on the average in terms of area and 10.5$\mathbf{3 6 . 4 \%}$ in terms of performance delay.
\end{abstract}

KEYWORD-Residue Number System (RNS), Modulo, Multiplier

\section{I . INTRODUCTION}

Residue number systems (RNS) are a good alternative to the conventional arithmetic, based on a weighted number system. The most common moduli sets used in RNS applications are the traditional $\left\{2^{n}, 2^{n}-1,2^{n}+1\right\}$ set $[1,2$, 3]. Also, the novel $\left\{2^{2 n}-1,2^{n}, 2^{2 n}+1\right\}$ [4] moduli set, with a dynamic range of $5 n$ bit. Recently, a 4-modulus base set $\left\{2^{n}-1,2^{n}+3,2^{n}+1,2^{n}-3\right\}$ has been proposed [5] as a hierarchical base composed by two pairs of moduli [6]. Even though several moduli sets have been proposed using modulo $\left(2^{n}-3\right)$ operations, the design of the required modulo $\left(2^{n}-3\right)$ multipliers is still a key challenge. The improved units for modulo $\left(2^{n}-3\right)$ multipliers have been proposed in [7] and employ a modular compressor (4:1) modulo $\left(2^{n}-3\right)$ to implement the final addition. However, they are not very efficient.

In this paper, we propose an improved algorithm and architecture for modulo $\left(2^{n}-3\right)$ multiplication, which removes all the drawbacks of the state-of-art modulo $\left(2^{n}-3\right)$ multiplier by applying some simple correction terms. The proposed algorithm employs several simple operation units to replace the modular compressor (4:1) modulo $\left(2^{n}-3\right)$ used in [7].

\section{II . BASIC THEORY}

For an integer $X$, there is

$$
\langle X\rangle_{2^{n}-3}=x, \quad x \in\left[0,2^{n}-3\right)
$$

$\left[0,2^{n}-3\right)$ is defined as the legitimate range of modulus $\left(2^{n}-3\right)$. In other words, the residue set of modulus $\left(2^{n}-3\right)$ is $\left[0,2^{n}-3\right)$. There is a gap between the legitimate range of modulus $\left(2^{n}-3\right)$ and the representation range of a n-bit binary representation. Through our research, we found that if we can bridge this gap and extend the residue set of modulus $\left(2^{n}-3\right)$ from $\left[0,2^{n}-3\right)$ to $\left[0,2^{n}-1\right]$, it will simply the corresponding operations. Because the value of 0 can be expressed as $2^{n}-3$ and 0 , the value 1 as $2^{n}-2$ and 1 , the value of 2 as $2^{n}-1$ and 2 . Thus, the residue set of modulus $\left(2^{n}-3\right)$ is extended from $\left[0,2^{n}-3\right)$ to $\left[0,2^{n}-1\right]$ and it does not affect the computation results.

$$
\begin{aligned}
& \left\langle 2^{n}-3\right\rangle_{2^{n}-3}=0 \\
& \left\langle 2^{n}-2\right\rangle_{2^{n}-3}=1 \\
& \left\langle 2^{n}-1\right\rangle_{2^{n}-3}=2
\end{aligned}
$$

Before extending, there is

$$
\langle A+B\rangle_{2^{n}-3}=\left\{\begin{array}{cc}
A+B+3 & A+B \geq 2^{n}-3 \\
A+B & A+B \geq 2^{n}-3
\end{array}\right.
$$


After extending, we have

$$
\langle A+B\rangle_{2^{n}-3}=\left\{\begin{array}{cc}
A+B+3 & A+B \geq 2^{n} \\
A+B & A+B \geq 2^{n}
\end{array}\right.
$$

and we just need to cope with the bits weighted by $2^{n+k}(k \geq 0)$. The process to cope with the bits weighted by $2^{n+k}(k \geq 0)$ as shown in (7) and (8) by applying a simple correction is very simple:

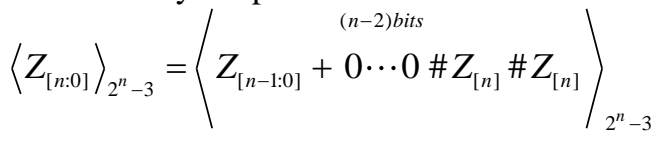

And

$$
\begin{aligned}
& \left\langle Z_{[2 n-1: 0]}\right\rangle_{2^{n}-3}=\left\langle Z_{[n-1: 0]}+3 Z_{[2 n-1: n]}\right\rangle_{2^{n}-3} \\
& =\left\langle Z_{[n-1: 0]}+Z_{[2 n-1: n]}+Z_{[2 n-2: n \# \# 2 n-1]}+0 \cdots 0 \# Z_{[2 n-1]} \# 0\right\rangle
\end{aligned}
$$

Where $Z_{[w: v]}$ represents bits of $Z$ originally located in positions from $v$ (less significant) to $w$ (more significant) and the symbol \# is used to concatenate bits.

\section{The Proposed AlgoRithm}

Suppose that $A_{[n-1: 0]} \times B_{[n-1: 0]}=P_{[2 n-1: 0]}$ and modulo $\left(2^{n}-3\right)$ multiplication can be described as:

$$
\begin{aligned}
& \left\langle A_{[n-1: 0]} \times B_{[n-1: 0]}\right\rangle_{2^{n}-3}=\left\langle P_{[2 n-1: 0]}\right\rangle_{2^{n}-3} \\
& =\left\langle P_{[n-1: 0]}+3 P_{[2 n-1: n]}\right\rangle_{2^{n}-3} \\
& =\left\langle P_{[n-1: 0]}+P_{[2 n-1: n]}+P_{[2 n-2: n \# 2 n-1]}+0 \cdots 0 \# P_{[2 n-1]} \# 0\right\rangle_{2^{n}-3}
\end{aligned}
$$

The former 3 terms $P_{[n-1: 0]}, P_{[2 n-1: n]}$ and $P_{[2 n-2: n \# 2 n-1]}$ in (9) can be computed with one n-bit CSA (Carry Save Adder) structure at first. The fourth term $0 \cdots 0 \# P_{[2 n-1]} \# 0$ just has one bit data in the second bit and is very difficult to merge with the former 3 terms $P_{[n-1: 0]} P_{[2 n-1: n]}$ and $P_{[2 n-2: n \# 2 n-1]}$. And it is reserved for mergence with some other term produced in the latter computation.

$$
P_{[n-1: 0]}+P_{[2 n-1: n]}+P_{[2 n-2: n \# 2 n-1]} \stackrel{C S A}{\longrightarrow} L_{[n-1: 0]}+2 H_{[n-1: 0]}
$$

Where ${ }^{L_{n-1: 0]}}$ and $H_{[n-1: 0]}$ are sum output data and carry output data of the n-bit CSA structure, respectively. And (10) can be rewritten as:

$$
\begin{aligned}
& \left\langle L_{[n-1: 0]}+2 H_{[n-1: 0]}\right\rangle_{2^{n}-3}=\left\langle L_{[n-1: 0]}+H_{[n-1: 0]} \# 0\right\rangle_{2^{n}-3} \\
& =\left\langle\left.\begin{array}{c}
(n-2) b i t s \\
L_{[n-1: 0]}+H_{[n-2: 0 \# n-1]}+0 \cdots 0 \# H_{[n-1]} \# 0
\end{array}\right|_{2^{n}-3} ^{(n-1) b i t s}\right. \\
& =\left\langle\left.\begin{array}{c}
(n-1) \text { bits } \\
L_{[n-1: 0]}+H_{[n-2: 0 \# n-1]}+0 \cdots 0 \# H_{[n-1]}+0 \cdots 0 \# H_{[n-1]}
\end{array}\right|_{2^{n}-3(11)}\right.
\end{aligned}
$$

The former 3 terms in (11) can be computed using one nbit binary adder with $L_{[n-1: 0]}$ and $H_{[n-2: 0 \# n-1]}$ as the two addends and $H_{[n-1]}$ as the carry-in and $R_{[n: 0]}$ is the sum of the former 3 terms in (11). And the fourth term in (11) just has one bit data in the first bit and is easy to merge with the fourth term in (9). It is reserved for mergence with the fourth term in (9).

$$
L_{[n-1: 0]}+H_{[n-2: 0 \# n-1]}+0 \cdots 0 \# H_{[n-1]}=R_{[n: 0]}
$$

And (12) can be rewritten as:

$$
\left\langle R_{[n: 0]}\right\rangle_{2^{n}-3}=\left\langle R_{[n-1: 0]}+\stackrel{(n-2) \text { bits }}{0 \cdots 0 \# R_{[n]} \# R_{[n]}}\right\rangle_{2^{n}-3}
$$

The fourth terms in (9) and (11) can be merged as $(n-2)$ bits

$0 \cdots 0 \# P_{[2 n-1]} \# H_{[n-1]}$. One 2-bit binary adder is used to $(n-2)$ bits $\quad(n-2)$ bits

compute $\quad 0 \cdots 0 \# P_{[2 n-1]} \# H_{[n-1]}+0 \cdots 0 \# R_{[n]} \# R_{[n]} \quad$ and (n-3)bits

$0 \cdots 0 \# G_{[2: 0]}$ is the result as:

$$
\begin{aligned}
& (n-2) \text { bits } \quad(n-2) \text { bits } \quad(n-3) \text { bits } \\
& 0 \cdots 0 \# P_{[2 n-1]} \# H_{[n-1]}+0 \cdots 0 \# R_{[n]} \# R_{[n]}=0 \cdots 0 \# G_{[2: 0]}
\end{aligned}
$$

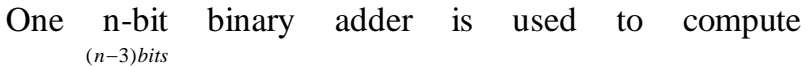
$R_{[n-1: 0]}+0 \cdots 0 \# G_{[2: 0]}$ and $Q_{[n: 0]}$ is the sum:

$$
R_{[n-1: 0]}+0 \cdots 0 \# G_{[2: 0]}=Q_{[n: 0]}
$$

Because $Q_{[n: 0]}$ has $\mathrm{n}+1$ bits, we have to make a simple correction as:

$$
\left\langle Q_{[n: 0]}\right\rangle_{2^{n}-3}=\left\langle Q_{[n-1: 0]}+\begin{array}{c}
(n-2) \text { bits } \\
0 \cdots 0 \# Q_{[n]} \# Q_{[n]}
\end{array}\right\rangle_{2^{n}-3}
$$




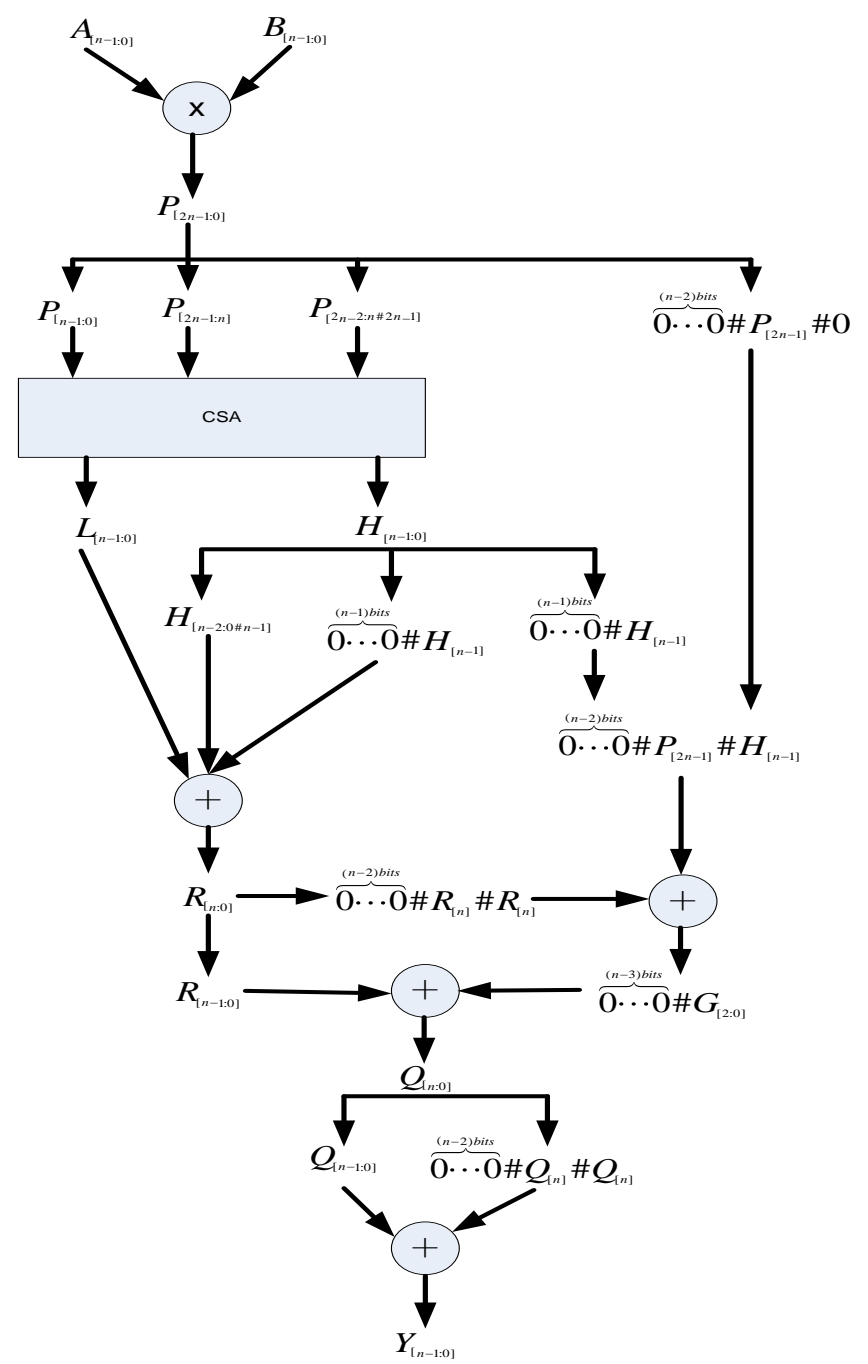

Fig.1. Proposed Architecture for Modulo $\left(2^{n}-3\right)$ Multiplication

The final result is $Y_{[n-1: 0]}$ :

$$
Q_{[n-1: 0]}+0 \cdots 0 \# Q_{[n]} \# Q_{[n]}=Y_{[n-1: 0]}
$$

$Y_{[n-1: 0]}$ in (17) will not overflow and the corresponding proof is given as:

Since $A_{[n-1: 0]} \times B_{[n-1: 0]}=P_{[2 n-1: 0]}$, it is impossible that all the bits of $P_{[2 n-1: 0]}$ are 1. Therefore it can be assumed that the largest value of $R_{[n: 0]}$ is $2^{n+1}-2$ from (9)-(12). Let us take the worst case, that is $R_{[n: 0]}=2^{n+1}-2, P_{[2 n-1]}=1$ and $H_{[n-1]}=1$. From (14) and (15), we get $Q_{[n: 0]}=2^{n}+4$. And $Y_{[n-1: 0]}$ can be gotten as $Y_{[n-1: 0]}=7$. Obviously, if $2^{n}-1 \geq 7$, that is $n \geq 3, Y_{[n-1: 0]}$ will not overflow. In fact, when $n<3$, there is just $n=2$ and $2^{n}-3=1$. It is of no significance for
RRNS. Therefore, there is no overflow of $Y_{[n-1: 0]}$ in (17).

Fig. 1 plots the proposed architecture for modulo $\left(2^{n}-3\right)$ multiplication.

\section{ANALYSIS AND COMPARISON}

The proposed modulo $\left(2^{n}-3\right)$ multiplier is composed of a binary multiplier, a n-bit CSA, a n-bit binary adder, a 2-bit binary adder and two n-bit binary adders. The multiplier in [5] is composed of a binary multiplier and a modular compressor (4:1) modulo $\left(2^{n}-3\right)$. The modular compressor (4:1) modulo $\left(2^{n}-3\right)$ is very complex and consists of 4 n-bit binary adders, $1(n+1)$-bit binary adder, 2 n-bit (2:1) MUXs and 2 control logic modules, which contains two 2 ANDs and 2 (2:1) MUXs. The conventional modulo $\left(2^{n}-3\right)$ multiplier is implemented by Synopsys DesignWare [6], which is expressed as "Conv" in Table I . It is composed of a binary multiplier, (n-1) n-bit binary adders, (n-1) n-bit (2:1) MUXs and (n-1) control logic modules, which are same with that of [5]. Table I lists hardware comparison results.

TABLE I . HARDWARE COMPARISON RESULTS

\begin{tabular}{|c|c|c|c|}
\hline & Conv & {$[5]$} & Proposed \\
\hline$n \times n$ multiplier & 1 & 1 & 1 \\
\hline n-bit CSA & - & - & 1 \\
\hline n-bit adder & $\mathrm{n}-1$ & 4 & 3 \\
\hline$(\mathrm{n}+1)$ bit adder & - & 1 & - \\
\hline n-bit (2:1) MUX & $\mathrm{n}-1$ & 2 & - \\
\hline Control logic & $\mathrm{n}-1$ & 2 & - \\
\hline 2-bit adder & - & - & 1 \\
\hline
\end{tabular}

Based on a column compression tree multiplier [9], a binary multiplier requires $3\left\lceil\log _{2} n\right\rceil$ units of time of a single-bit full adder, where $\left.\Gamma^{*}\right\rceil$ is the integer greater than or equal to $*$. An n-bit binary adder requires $\left\lceil\log _{2} n\right\rceil+1$ units of time [9], [10]. The delay of the CSA is 1 units of time. A (2:1) MUX has the delay of 1 units of time. It can be assumed that the control logic used in the conventional modulo $\left(2^{n}-3\right)$ multipliers and the modulo $\left(2^{n}-3\right)$ multipliers in [7] has 2 units of time, because it has two (2:1) MUXs in the critical path. Therefore,

$$
\begin{gathered}
T_{\text {Pro }}=T_{\text {mult }}+T_{C S A}+3 T_{\text {adder }, n}+T_{\text {adder }, 2} \\
=6\left\lceil\log _{2} n\right\rceil+6 \\
T_{[5]}=T_{\text {mult }}+3 T_{\text {adder }, n}+T_{\text {adder }, n+1}+2 T_{\text {control }}+2 T_{M U X} \\
=6\left\lceil\log _{2} n\right\rceil+\left\lceil\log _{2}(n+1)\right\rceil+10
\end{gathered}
$$




$$
\begin{aligned}
T_{\text {Conv }} & =T_{\text {mult }}+(n-1) T_{\text {adder }, n}+(n-1) T_{\text {control }}+(n-1) T_{M U X} \\
& =(n+2)\left\lceil\log _{2} n\right\rceil+3 n-3
\end{aligned}
$$

The fast modulo $\left(2^{n}-3\right)$ multiplier proposed in this paper was modeled in structural Verilog HDL for a general value of $\mathrm{n}$ and its operation was exhaustively verified. The fast modulo $\left(2^{n}-3\right)$ multiplier proposed in this paper was implemented using 90nm CMOS process technology. The Synopsys Design Compiler tool version Y-2006.06-SP4 was used to get the synthesized results. During synthesis, all the designs were optimized for speed and, as a secondary target, and the tool was instructed to try to recover as much area as possible. The obtained results are plotted in Fig.2 and Fig. 3.

The synthesized results demonstrate that the proposed multipliers can achieve up to $36.4 \%$ and $71.4 \%$ faster designs than the conventional modulo $\left(2^{n}-3\right)$ multipliers and the modulo $\left(2^{n}-3\right)$ multipliers in [5], respectively. On the average, the proposed multipliers are faster than the conventional modulo $\left(2^{n}-3\right)$ multipliers and the modulo $\left(2^{n}-3\right)$ multipliers in [5] by $25.8 \%$ and $47.0 \%$, respectively. The proposed multipliers are $3.9 \%$ and $33.1 \%$ more area efficient than the conventional modulo $\left(2^{n}-3\right)$ multipliers and the modulo $\left(2^{n}-3\right)$ multipliers in [5] on the average. From the synthesized results, the proposed modulo $\left(2^{n}-3\right)$ multiplier can improve the current state of the art by $3.9 \%$ on the average in terms of area and $10.5-36.4 \%$ in terms of performance delay.

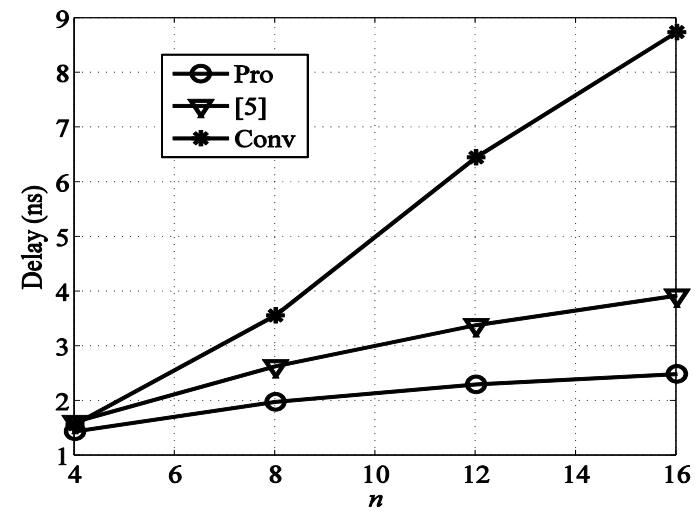

Fig.2. The Delay Performance of the Proposed Multipliers and the Reference Multipliers

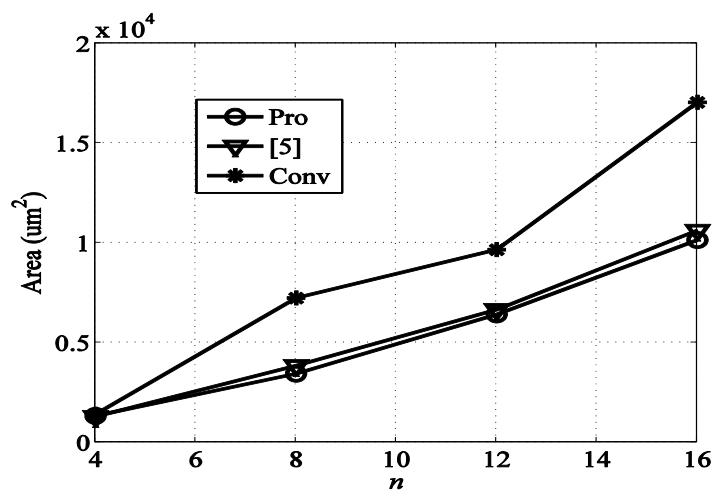

Fig.3. The Synthesized Area of the Proposed Multipliers and the Reference Multipliers

Considering the area $\times$ time 2 product, the radio of efficiency of the proposed multiplier to the reference multiplier can be defined as:

$$
E_{\text {Ref,Pro }}=A E_{\text {Ref,Pro }} \times T E_{\text {Ref,Pro }}^{2}=\frac{A_{\text {Ref }}}{A_{\text {Pro }}} \times\left(\frac{T_{\text {Ref }}}{T_{\text {Pro }}}\right)^{2}
$$

The ratios of efficiency of the proposed multipliers to the multipliers in [7] and the conventional modulo $\left(2^{n}-3\right)$ multipliers are $1.21-2.59$ and $1.26-20.58$, respectively.

\section{CONCLUSION}

In this paper, an improved algorithm for designing efficient modulo $\left(2^{n}-3\right)$ multipliers is proposed. By applying some simple correction terms, the proposed multiplier is the most efficient among all the known modulo $\left(2^{n}-3\right)$ multipliers. The synthesized results demonstrate that the proposed modulo $\left(2^{n}-3\right)$ multiplier can improve the current state of the art by $3.9 \%$ on the average in terms of area and $10.5-36.4 \%$ in terms of performance delay.

\section{REFERENCES}

[1] F. E. P. Dale Gallaher and P. Srinivasan, "The digit paralell method for fast $r n$ to weighted number system conversion for specific moduli $(2 \mathrm{n}-1,2 \mathrm{n}, 2 \mathrm{n}+1)$," IEEE Transactions on Circuits and Systems - II: Analog and Digital Signal Processing, 1997.

[2] L.Li, J.Hu, and Y. Chen. "Modified Booth encoding modulo (2n-1) multipliers," IEICE Electron. Express, vol. 9, no.5, pp. 352-358, March 2012.

[3] T.-B. Juang, C.-C. Chiu, and M.-Y. Tsai, "Improved Area-Efficient Weighted Modulo 2n+1 Adder Design With Simple Correction Schemes," IEEE Trans. Circuits Syst. II, Exp. Briefs, vol.57, pp. 198-202, March 2010.

[4] A. Hariri, K. Navi, and R. Rastegar, "A new high dynamic range moduli set with efficient reverse converter," Computers and Mathematics with Applications, vol. 55, no. 4, pp. $660-668,2008$.

[5] M.-H. Sheu, S.-H. Lin, C. Chen, and S.-W. Yang, "An efficient vlsi design for a residue to binary converter for general balance moduli $(2 \mathrm{n}-3,2 \mathrm{n}+1,2 \mathrm{n}-1,2 \mathrm{n}+3)$," Circuits and Systems II: Express Briefs, IEEE Transactions on, vol. 51, no. 3, pp. 152 - 155, march 2004.

[6] L.-S. Didier and P.-Y. Rivaille, "A generalization of a fast rns conversion for a new 4-modulus base," Circuits and Systems II: Express Briefs, IEEE Transactions on, vol. 56, no. 1, pp. 46 -50, Jan. 2009. 
[7] P. M. Matutino, R. Chaves, L. Sousa, "Arithmetic units for RNS moduli $\{2 n-3\}$ and $\{2 n+3\}$ operations", 13th Euromicro Conf. Digital System Design: Architectures, Methods and Tools, pp.243-246, 2010.

[8] Synopsys, DesignWare datasheets, Version Y-2006.06
[9] B. Sinha and P. Srimani, "Fast Parallel Algorithms for Binary Multiplication and Their Implementation on Systolic Architectures," IEEE Trans. Computers, vol. 38, no. 3, pp. 424-431, Mar. 1989.

[10] T. Herman, "Linear Algorithms That Are Efficiently Parallelized to Time O(log n)," Technical Report TR-85-17, Dept. of Computer Science, Univ. of Texas at Austin, Sept. 1985. 